\title{
Percepções de Profissionais sobre Atendimentos em Saúde para Mulheres Usuárias de Crack
}

\author{
Luísa Maciel ${ }^{1} \quad$ Daniela Chambart ${ }^{1}$ \\ ${ }^{\text {P}}$ Pontifícia Universidade Católica do Rio Grande do Sul, ${ }^{\text {} P o n t i f i ́ c i a ~ U n i v e r s i d a d e ~ C a t o ́ l i c a ~ d o ~ R i o ~ G r a n d e ~ d o ~ S u l, ~}$ \\ RS, Brasil. \\ RS, Brasil. \\ Jaluza Aimèe Schneider ${ }^{1}$ \\ ${ }^{1}$ Pontifícia Universidade Católica do Rio Grande do Sul, \\ RS, Brasil. \\ Rodrigo Grassi-Oliveira ${ }^{1}$ \\ ${ }^{1}$ Pontifícia Universidade Católica do Rio Grande do Sul, \\ RS, Brasil. \\ Luísa Fernanda Habigzang ${ }^{1}$ \\ ${ }^{1}$ Pontifícia Universidade Católica do Rio Grande do Sul, RS, Brasil.
}

Resumo: As percepções dos profissionais de saúde que atendem mulheres usuárias de crack podem auxiliar no aprimoramento dos atendimentos. Assim, este estudo objetivou compreender essas percepções. Se trata de um estudo qualitativo, com 33 participantes divididos em três grupos focais submetidos à análise temática. Estabeleceram-se dois eixos centrais: a) usuárias de crack e b) serviços de saúde. Posteriormente, identificaram-se oito temas e seis subtemas. No primeiro eixo destacaram-se: características das usuárias, ausência de rede de apoio, violência, estigma de gênero, prostituição e comorbidades clínicas. No segundo ressaltaram-se as dificuldades na articulação entre os serviços e questões a serem aprimoradas, como: prevenção na atenção básica, motivação dos profissionais, serviços específicos para mulheres, especificidades da gestação e combate ao estigma. A partir dos resultados, foram identificados e discutidos os desafios e as potencialidades que se dão nos atendimentos de mulheres usuárias de crack.

Palavras-chave: Saúde Pública, Profissionais da Saúde, Transtorno por Uso de Substâncias, Crack, Mulheres.

\section{Perceptions of Professionals about Health Care for Women Crack Users}

\begin{abstract}
The perceptions of health professionals that serve crack-using women can help in the improvement of health care. Thus, this study verified such perceptions via a qualitative study with 33 participants, divided into three focus groups subjected to thematic analysis. Two a priori central axes were defined: 1) crack-using women, and 2) health services. Subsequently, eight themes and six subthemes were identified. Axis 1) showed: characteristics of the users, lack of support network, violence, gender stigma, prostitution and clinical comorbidities. In axis 2), there were challenges in articulating services and issues to be improved, such as prevention in primary care, motivation of professionals, specific services for women, specific aspects of gestation and stigma. The results allowed to identify and discuss the potentialities and challenges in providing healthcare services to crack-using women.
\end{abstract}

Keywords: Public Health, Health Professionals, Substance use Disorder, Crack, Women. 


\title{
Percepciones de Profesionales sobre Atenciones en Salud para Mujeres Usuarias de Crack
}

\begin{abstract}
Resumen: Las percepciones de los profesionales de la salud que atienden a mujeres usuarias de crack pueden auxiliar en el perfeccionamiento de las atenciones. Este estudio objetivó comprender las percepciones de profesionales de la salud sobre atenciones a las usuarias de crack. Es un estudio cualitativo, con 33 participantes, divididos en tres grupos focales sometidos al análisis temático. Dos ejes centrales se establecieron a priori: 1) mujeres usuarias de crack; y 2) servicios de salud. Posteriormente, se identificaron ocho temas y seis subtemas. En el primer eje se destacaron: las características de las usuarias, la ausencia de la red de apoyo, la violencia, el estigma de género, la prostitución y las comorbilidades clínicas. En el segundo eje, se resalta las dificultades en la articulación entre los servicios y también cuestiones a ser mejoradas, como: prevención en la atención básica, motivación de los profesionales, servicios específicos para mujeres, especificidades de la gestación y combate al estigma. A partir de los resultados fue posible identificar y discutir las potencialidades y desafíos en las atenciones de mujeres usuarias de crack.
\end{abstract}

Palabras clave: Salud Pública, Profesionales de la Salud, Trastorno por Uso de Sustancias, Crack, Mujeres.

O uso de crack no Brasil é um problema de saúde pública, considerando as altas taxas de consumo entre a população (Secretária Nacional de Políticas sobre Drogas, 2013; United Nations Office on Drugs and Crime, 2016). De acordo com o segundo Levantamento Nacional de Álcool e Drogas (Lenad), aproximadamente 1,7 milhões da população brasileira já fizeram uso de crack pelo menos uma vez na vida (Laranjeira et al., 2012). De acordo com o relatório de Bastos e Bertoni (2014), em torno de 20\% dos usuários de crack no Brasil são mulheres. Porém destaca-se que as usuárias tendem a não buscar os serviços de saúde para o tratamento especializado. Dentre os fatores que contribuem para a menor procura de atendimento entre as mulheres estão o sentimento de vergonha e medo do julgamento social e até dos próprios profissionais que as atendem, além da falta da inclusão das especificidades de gênero no tratamento (Cruz et al., 2014; Limberger, Schneider, \& Andretta 2015). Entre as principais especificidades para o tratamento de usuárias de substâncias, a literatura indica a inclusão de intervenções que contemplem desigualdades sociais e de gênero, como por exemplo a ampliação do acesso ao contexto profissional, a diminuição de risco de contrair Infecções Sexualmente Transmissíveis (ISTs) e de ser vítima de qualquer tipo de violência, além de abordar aspectos sobre a maternidade (Bertoni et al., 2014; Gueta, 2014; Guimarães et al., 2017; United Nations Office on Drugs and Crime, 2016).

Considerando as consequências significativas associadas ao consumo de crack em nível interpessoal, psiquíco, comportamental e físico, há a necessidade de desenvolvimento e aprimoramento de programas no Sistema Único de Saúde (SUS), de acordo com os seus principios de universalidade, equidade e integralidade, visando estratégias adequadas para o tratamento do usuário de substância (Secretaria Nacional de Políticas sobre Drogas, 2013). Assim, atualmente, o Brasil conta com políticas públicas no contexto do consumo de substâncias, como a Política de Atenção Integral ao Usuário de Álcool e Outras Drogas (Brasil, 2005), que visa atuar dentro dos princípios do SUS, descritos anteriormente, considerando as especificidades dessa população. Ainda, tal política visa fortalecer a rede, promover atenção integral aos usuários e garantir acesso fácil para a utilização de serviços na rede de atenção à saúde mental alternativos aos hospitais psiquiátricos, como, por exemplo, os Centros de Atenção Psicossocial (CAPS) e as Unidades de Acolhimento (UA). A Política Nacional Sobre Drogas tem como objetivo reduzir a oferta e a demanda de drogas nos país, por meio de ações de prevenção, 
educação, redução de danos e ações para minimizar o tráfico (Brasil, 2005). Além disso, visando construir estratégias específicas para o crack, implementou-se o Plano Integrado de Enfrentamento ao Crack e Outras Drogas (Brasil, 2010), prevendo mais leitos hospitalares para essa população, a ampliação da rede de atenção ao usuário de crack e o incentivo ao desenvolvimento de capacitações para os profissionais.

Apesar das ações e estratégias descritas nas políticas públicas brasileiras serem adequadas para os usuários de crack, observam-se dificuldades para a sua realização nos serviços especializados (Schneider \& Lima, 2011). Entre as dificuldades neste contexto, se destacam a rotina desgastante dos profissionais (e.g. falta de recursos financeiros e número elevado de atendimentos por dia) e a falta de capacitações e materiais diretivos com informações sobre as especificidades dos usuários (Costa \& Paiva, 2016; Schneider \& Lima, 2011). Destaca-se que, em relação à população feminina usuária de crack, as informações e estratégias são ainda mais carentes. As cartilhas nacionais sobre o transtorno por uso de substâncias e as políticas de atenção integral aos usuários de crack não discutem as especificidades de gênero. Tal fato tende a reforçar a prática de intervenções baseada nas percepções e crenças pessoais, incluindo julgamentos e preconceitos relacionados às mulheres usuária de drogas (Bastos \& Bertoni, 2014; Limberger et al., 2015). Além disso, o manejo inadequado dos profissionais com as usuárias pode contribuir para a baixa adesão ao tratamento por essas (Ferreira, Borba, Capistrano, Czarnobay, \& Maftum, 2015; Salles \& Silva, 2017).

O relatório das Nações Unidas (International Narcotics Control Bourd, 2017) ressalta a urgência da inclusão de especificidades de gênero nas políticas públicas e implementação de tratamento para a população usuária de drogas. A complexidade envolvida, na maioria dos casos dos atendimentos de usuários, ressalta a necessidade de os profissionais obterem conhecimento sobre o tema (Laport, Costa, Mota, \& Ronzani, 2016). Estudos anteriores demonstram que profissionais que atuam na rede de saúde e assistência social de mulheres usuárias de crack tendem a reproduzir o discurso baseado no esteriótipo de gênero, como associar a mulher a papéis de passividade e de cuidadoras da família (Oliveira, Paiva, \& Valente, 2006; Silva, Pereira, \& Penna, 2018). Além disso, é indicado que os profissionais que atuam com tal população tenham capacitações que contemplem as demandas do transtorno por uso de substâncias, com informações para a atuação efetiva e aumento de competência para lidar com as adversidades do trabalho (Barbosa, Cobayashi, \& Oliveira, 2017; Ferreira et al., 2015). Assim, buscar estratégias para qualificar os profissionais e melhorar suas condições de trabalho pode diminuir o nível de sobrecarga emocional dos mesmos (Horta, Esswein, \& Horta, 2013).

Destaca-se também a necessidade de se considerar diferenças biopsicossociais de homens e mulheres usuários de crack, construindo atendimentos considerando as vulnerabilidades psicossociais relacionadas ao gênero feminino, tais como maior risco de se envolver com prostituição, maior probabilidade de ser portadora de Vírus da Imunodeficiência Humana (HIV) e de demais ISTs, índices elevados de comorbidades psiquiátricas, ter experienciado eventos traumáticos e ter sido vítima de violência ao longo da vida (Bertoni, et al., 2014; Grassi-Oliveira, et al., 2012; Guimarães et al., 2017; Limberger, Nascimento, Schneider, \& Andretta, 2016). Diante deste contexto, é evidente a importância de capacitações para aumentar a efetividade dos atendimentos de usuárias de crack e para diminuir o impacto do estresse pelas diferentes demandas que dificultam os atendimentos (Salles \& Silva, 2017; Silva et al., 2018). Para a realização de capacitações específicas, é necessário que os profissionais sejam ouvidos e possam indicar aspectos positivos e desafios de seu cotidiano de trabalho. Nesta perspectiva, o objetivo desse estudo foi compreender as percepções dos profissionais de saúde sobre a rede de atendimento em saúde pública para mulheres usuárias de crack. Buscou-se investigar as percepções e crenças dos profissionais sobre usuárias de crack e as especificidades dos atendimentos a esta população.

\section{Método}

\section{Participantes}

Participaram deste estudo 33 profissionais, todos atuantes em serviços de saúde. que foram divididos em três Grupos Focais (GF). Dois GFs foram realizados na cidade de Porto Alegre e o outro foi realizado em São Paulo. Os participantes foram selecionados por conveniência e, como critério de inclusão para a participação, eles deveriam ter atuação na rede pública no atendimento de usuárias de crack pelo período mínimo 
de um ano. Estes atuavam em Postos de Saúde (PS), Centros de Atenção Psicossocial Álcool e Drogas (CAPS $\mathrm{AD})$ e hospitais de referência para internação.

A amostra foi composta predominantemente por mulheres $(82,4 \%)$, com idade média de 35,1 anos $(\mathrm{DP}=8,7)$. A maioria possuía formação superior (75,8\%). Os profissionais eram formados em Serviço Social $(28,6 \%)$, Enfermagem (21,4\%), Técnico em Enfermagem (14,3\%), Psicologia (17,8\%) e Psiquiatria $(17,8 \%)$. O tempo médio de atuação com usuários de substâncias, incluindo o período de estágio durante a graduação, foi de 4,5 anos (DP = 3,6), e o tempo médio de atuação dos participantes especificamente com mulheres usuárias foi de 3,9 anos ( $\mathrm{DP}=3,3$ ).

\section{Instrumentos}

Os instrumentos utilizados foram: ficha de dados sociodemográficos e roteiro para discussão nos GFs com 11 questões norteadoras. As questões investigaram os seguintes tópicos: a) características das mulheres usuárias de crack; b) rotina de atendimento prestado às usuárias de crack; c) elementos que se diferenciam no atendimento a mulheres que consomem outros tipos de substâncias; d) elementos que diferenciam o atendimento a mulheres usuárias de crack comparado aos homens; e) desafios identificados no atendimento a usuárias de crack; f) autopercepção dos profissionais frente aos desafios; g) avaliação dos profissionais sobre os atendimentos do sistema de saúde para as mulheres usuárias de crack; h) sugestões de melhorias no serviço prestado; i) avaliação do fluxo de encaminhamentos; j) conhecimentos necessários para atender mulheres usuárias de crack; k) sugestões de conteúdos para um manual de capacitação profissional para atuar com mulheres usuárias de crack.

\section{Procedimentos de coleta}

O presente estudo faz parte de um projeto mais amplo com os seguintes objetivos: a) compreender as percepções das usuárias e de profissionais sobre serviços de saúde para mulheres usuárias de crack e b) desenvolver um manual para capacitação de profissionais da saúde que atendem a essa população. O projeto de pesquisa foi aprovado pelo Comitê de Ética em Pesquisa (CEP) da Pontifícia Universidade Católica do Rio Grande do Sul (PUCRS), sob o número 984.015.
Após aprovação do CEP, as instituições gestoras dos serviços de saúde referência para o atendimento de álcool e drogas de Porto Alegre foram contatadas a fim de se estabelecer parcerias para a coleta de dados. Foram realizadas parcerias com duas instituições, as quais assinaram o termo de concordância institucional. Em seguida, o estudo foi divulgado pelos gestores em suas equipes de trabalho. Também foi realizado o contato e estabelecimento da parceria com o Centro de Referência de Álcool, Tabaco e Outras Drogas (Cratod) em São Paulo. Os profissionais que preencheram os critérios de inclusão e que concordaram em participar voluntariamente do estudo assinaram um Termo de Consentimento Livre e Esclarecido (TCLE).

Como já fito, foram realizados três GFs: dois em Porto Alegre, nas dependências da universidade, e um em São Paulo, realizado no próprio Cratod. Todos os GFs tiveram apenas um encontro, com duração entre 90 e 120 minutos. Os Grupos foram coordenados pela mesma pesquisadora e contaram com a presença de um observador para anotar e indicar verbalizações e expressões não verbais, e um auxiliar para ajudar nas situações pontuais como, por exemplo, programar a câmera de vídeo. A discussão dos GFs foi gravada em áudio e vídeo; posteriormente, foi também transcrita.

\section{Análise de dados}

As gravações dos GFs, em áudio e vídeo, foram transcritas por dois auxiliares de pesquisa previamente treinados, e, posteriormente, foram revisados pela pesquisadora responsável deste estudo. Foram atribuídos códigos específicos para cada GF (e.g., GF 1, GF 2 e GF 3) e para cada participante (e.g., P1, P2 e P3) a fim de manter $\mathrm{o}$ anonimato dos profissionais participantes.

Os dados advindos dos GFs foram analisados a partir da análise temática proposta por Braun e Clarke (2006), com as seguintes etapas: a) familiarização com o material por meio de leituras da transcrição e anotações sobre o conteúdo; b) identificação e seleção de extratos para criação de temas adequados aos objetivos do estudo; c) revisão dos temas, verificando se os estratos estavam adequadamente alocados; d) refinamento da análise por meio da identificação indutiva de subtemas; e) revisão dos temas e subtemas por dois juízes independentes; f) comparação das análises feitas pelos juízes independentes com a análise prévia para verificar discrepâncias e possíveis reformulações 
dos temas e subtemas; g) discussão com os juízes acerca das divergências, visando consenso; h) finalização de temas e subtemas com exemplos representativos; e i) redação dos resultados.

\section{Resultados e discussão}

A partir da análise temática emergiram sete temas e seis subtemas organizados em dois eixos principais estabelecidos a priori, de acordo com os objetivos do estudo, denominados: a) "Mulheres usuárias de crack" e b) "Atendimento e serviços de saúde pública". No primeiro eixo, "Mulheres usuárias de crack", emergiram três temas e seis subtemas. No segundo eixo, "Atendimento e serviços de saúde pública", emergiram quatro temas. Os temas e subtemas de ambos os eixos e exemplos de unidades de análise (frases) estão apresentados nas Tabelas 1 e 2, respectivamente.

Tabela 1

Eixo temático "Mulheres usuárias de crack"

\begin{tabular}{|c|c|c|}
\hline Temas & Subtemas & Unidade de Análise Representativa \\
\hline $\begin{array}{l}\text { "Fotografia" das } \\
\text { mulheres }\end{array}$ & & $\begin{array}{l}\text { "Mulheres em situações de vulnerabilidade biopsicossocial. . . . Desempregadas, } \\
\text {... apresentam comportamento sexual de risco ou promiscuidade" (GF 1). } \\
\text { saixo nível de escolaridade. . . já possuem filhos e esses se encontram em } \\
\text { situaço de institucionalização ou são criados por outros familiares" (GF 2). }\end{array}$ \\
\hline \multirow[t]{3}{*}{ Rede de apoio } & & $\begin{array}{l}\text { "Enquanto as mulheres. ... estão em abuso intenso e precisam mais de ajuda, } \\
\text { elas são colocadas para fora e ficam sem nenhum apoio da família. Eu acho } \\
\text { que posso afirmar que nunca tive uma paciente que teve o auxílio da família } \\
\text { para fazer seu tratamento e que foi cuidada" (GF 1). }\end{array}$ \\
\hline & Gênero & $\begin{array}{l}\text { “Talvez seja diferente entre homens e mulheres, talvez a sociedade tenha uma } \\
\text { vontade maior de ajudar o homem dependente químico. . . talvez esse estigma } \\
\text { faça a mulher demorar mais para buscar atendimento. . . . Acho que é um } \\
\text { preconceito do perfil da mulher, do estigma. Então agora que eu to cheia de filho } \\
\text { para cuidar, to cheia de tarefa de casa pra fazer, e ninguém dá a mínima para } \\
\text { mim, se eu ainda disser que estou usando drogas, dai vai cair o mundo, vou ser } \\
\text { a desqualificada, a irresponsável, dai não vai ter para mim” (GF 1). }\end{array}$ \\
\hline & Violência & $\begin{array}{l}\text { "Tem várias pacientes minhas que espontaneamente trazem o histórico de } \\
\text { violência sexual, várias violências, da família ser violenta" (GF 1). } \\
\text { "Era casada com um companheiro que comandava o tráfico, ou fazia alguma } \\
\text { coisa junto ao tráfico e a colocava pra trabalhar ou vender ou pra se prostituir, } \\
\text { uma série de coisas. Casos muito graves de violência doméstica" (GF 2). }\end{array}$ \\
\hline \multirow[t]{4}{*}{$\begin{array}{l}\text { Comorbidades e } \\
\text { consequências }\end{array}$} & $\begin{array}{l}\text { Comorbidades } \\
\text { clínicas e } \\
\text { psiquiátricas }\end{array}$ & $\begin{array}{l}\text { “Comorbidade com outras patologias psiquiátricas, transtorno de humor } \\
\text { bipolar, Borderline. . . . Além do HIV, também outras doenças clínicas: } \\
\text { tuberculose, DST, são muito vulneráveis a doenças infecciosas” (GF 2). }\end{array}$ \\
\hline & $\begin{array}{l}\text { Déficit } \\
\text { cognitivo }\end{array}$ & $\begin{array}{l}\text { “A limitação cognitiva é muito presente. Cognitivamente são bem } \\
\text { deterioradas" (GF 1). }\end{array}$ \\
\hline & $\begin{array}{l}\text { Deterioração } \\
\text { física }\end{array}$ & $\begin{array}{l}\text { “O aspecto físico né, muito deteriorado, a idade aparente é superior à } \\
\text { cronológica. .. . muitas vezes a questão dos dentes a gente vê... . questão de pele, } \\
\text { mais doentes. Então a gente vê que são visivelmente mais doentes"(GF 2). }\end{array}$ \\
\hline & Prostituição & $\begin{array}{l}\text { "Prostituição não como profissão, mas prostituição como decorrente do uso de } \\
\text { substância. . . . Na verdade elas se prostituem quando é o ultimo recurso. Elas } \\
\text { já perderam o emprego, não têm mais como ter o dinheiro para comprar a } \\
\text { substancia. Mais de 90\% já se prostituiu em algum momento"(GF 1). }\end{array}$ \\
\hline
\end{tabular}

\section{Mulheres usuárias de crack}

No eixo temático "Mulheres usuárias de crack" (Tabela 1) foram incluídos os temas referentes à visão dos profissionais sobre tal população a partir de suas experiências prévias. No primeiro tema deste eixo, denominado "Fotografia", foram observadas as percepções dos GFs sobre as principais características das usuárias de crack. As características identificadas foram: vulnerabilidade biopsicossocial, baixo nível de escolaridade, desemprego, início do uso da droga por meio de amigos ou companheiro, comportamento sexual de risco e ter filhos que se encontram em instituições ou que são criados por outros familiares. Os GFs concluíram que as mulheres usuárias de crack são uma população de extrema vulnerabilidade decorrente das condições sociais precárias em que a maioria se encontra. 
As características destacadas pelo GF estão de acordo com os estudos nacionais sobre o perfil de usuários de crack (Dualibi, Ribeiro, \& Laranjeira, 2008; Limberger, Nascimento, Schneider, \& Andretta, 2016). As percepções dos participantes indicaram que as usuárias de crack são casos complexos, como um "prato quebrado" (GF 2). Essas percepções revelaram a impotência sentida pelos profissionais diante de algumas demandas que extrapolam os cuidados de saúde. Além disso, o estigma social sobre uso do crack estimula estes casos como de díficil solução ou de prognóstico negativo, o que interfere no manejo dos profissionais em auxiliar efetivamente as usuárias.

O segundo tema "Rede de apoio", compilou as percepções dos profissionais sobre as relações de apoio e proteção existentes para as mulheres usuárias de crack. Os participantes dos GFs constataram que o apoio recebido por essa população é precário e os vínculos são caracterizados por violência, negligência, conflitos e falta de assistência familiar. A ausência de suporte é presente na história destas mulheres e a busca de tratamento ocorre por conta própria, sem auxílio ou amparo de outros. Estudos anteriores observaram a presença de vínculos familiares frágeis e a presença de histórico de violência dessas mulheres (Limberger et al., 2016). A ausência de apoio familiar tende a dificultar a busca e a adesão à ajuda especializada pelas usuárias de substâncias (Ferreira et al., 2015). A falta de suporte das mulheres usuárias de crack indica a necessidade de intervenções que favoreçam a construção de uma rede de apoio saudável (Ferreira et al., 2015; Büchele, Coelho, \& Lindner, 2009).

Ainda sobre o tema "Rede de apoio", foram identificados dois subtemas: "Gênero" e "Violência". Estes subtemas indicam fatores que foram compreendidos pelos profissionais como potencializadores de risco e vulnerabilidade relacionados ao suporte de tal população. Em relação ao "Gênero", os três GFs pontuaram que ser mulher implica na maior presença de estigma e preconceito por parte da família. A não realização de tarefas socialmente esperadas de acordo com estereótipos de gênero (e.g. cuidado com os filhos) aumenta o julgamento social sobre a incapacidade e irresponsabilidade das mulheres usuárias de substâncias (Cruz et al., 2014). O papel social das mulheres está associado a uma figura cuidadora, influenciando a diminuição de percepção da necessidade de cuidado por suas famílias e pela comunidade (Ramiro, Pandovani, \& Tucci, 2014). Observa-se que as expectativas sociais atreladas ao gênero podem influenciar fortemente a atuação dos profissionais com essa população na forma de reprodução do preconceito social, na manutenção do contexto de vulnerabilidade e vitimização, além da diminuição da probabilidade de implementação de intervenções que visem o empoderamento dessas mulheres sobre suas vidas, como, por exemplo, a inserção no mercado de trabalho e o aumento da autoestima (Moraes, Roso, \& Lara, 2018; Silva, 2018).

No subtema "Violência" foram identificadas descrições dos profissionais sobre o histórico de violência das mulheres atendidas. Todos os GFs apontaram que a presença de violência na infância e adolescência é comumente relatada pelas mulheres e que tais experiências ocorreram geralmente no contexto familiar. Os profissionais indicaram que tais mulheres, na vida adulta, são vítimas de violência perpetradas por clientes da prostituição e por parceiros íntimos. Os resultados encontrados corroboram a literatura sobre a associação entre histórico de violência na infância e uso de crack na vida adulta entre as mulheres (Smith, Homish, Leonard, \& Cornelius, 2012; Tractenberg et al., 2012; Vernaglia et al., 2017). Destaca-se que grande parte das mulheres que buscam os serviços de saúde não denuncia situações de violência. Considerando os altos índices de abuso entre essa população, é de extrema importância que os profissionais estejam capacitados para reconhecer sinais de violência e, assim, realizar a notificação e os encaminhamentos necessários para a proteção da mulher de forma humanizada e acolhedora (Oliveira, Rodrigues, Morais, \& Machado, 2016). Os serviços devem atuar como local de segurança e suporte para essas mulheres e, nesta perspectiva, é indicado que sejam específicos para a população feminina, diminuindo a possibilidade de novas revitimizações no próprio local de tratamento (United Nations Office on Drugs and Crime, 2016).

O terceiro tema identificado foi "Comorbidades e consequências". Esse tema foi dividido em quatro subtemas: "Comorbidades clínicas e psiquiátricas", "Déficit cognitivo", "Deterioração física” e "Prostituição". No subtema "Comorbidades clínicas e psiquiátricas" os participantes dos GFs apontaram a presença de ISTs - em especial o HIV - e comorbidades psiquiátricas entre as usuárias de crack. Dentre as comorbidades psiquiátricas observadas pelos profissionais, e também indicadas pela literatura, destacam-se o transtorno por uso de múltiplas 
substâncias, os transtornos de humor e o transtorno de personalidade Borderline (Dualibi et al., 2008; Ribeiro \& Laranjeira, 2012). As comorbidades psiquiátricas podem aumentar a insegurança dos profissionais, uma vez que colocam os mesmos na posição de lidar com sintomatologias diversificadas e graves. No que diz respeito às ISTs, o transtorno por uso de crack está altamente relacionado ao HIV e tal comorbidade está associada, por sua vez, à baixa adesão aos tratamentos indicados (Bastos \& Bertoni, 2014). O uso de drogas tende a aumentar a exposição a comportamentos sexuais de risco, observando-se uma relação entre tais comportamentos e o surgimento de ISTs (Guimarães et al., 2017; Vernaglia et al., 2017). Para as usuárias de drogas que possuem ISTs, há a necessidade da rede de saúde oferecer recursos e facilitações de acesso aos dois tratamentos (Pinto, Tancredi, Buchalla, \& Miranda, 2014).

Os GFs descreveram a dificuldade de compreensão e de insight - decorrentes do uso do crack - entre as mulheres usuárias, gerando o subtema "Déficit Cognitivo". Nesta perspectiva, um estudo identificou que mulheres usuárias de crack avaliam o risco frente a uma situação de forma distinta de não usuárias, com tomadas de decisão arriscadas e sem planejamento (Gladwin, Figner, Crone, \& Wiers, 2011). Os resultados de uma avaliação de tomadas de decisão de adolescentes e mulheres usuárias de crack verificou que ambos os grupos assumiram riscos e foram impulsivos de forma semelhante (Kluwe-Schiavon, Viola, Sanvicente-Vieira, Pezzi, \& Grassi-Oliveira, 2016). Assim, observa-se que determinadas funções cognitivas - que auxiliam na inibição de comportamentos de risco, percepção e processamento de informações - podem estar prejudicadas pelo abuso da substância (Gladwin et al., 2011). A presença de tais prejuízos requer, dos profissionais, habilidades para avaliar o comprometimento cognitivo e o padrão comportamental entre as usuárias de crack.

Os danos físicos perceptíveis pelo uso dessa substância se referem ao terceiro subtema, "Deterioração física”. Os GFs descreveram a má higiene das mulheres quando chegam aos serviços, além da presença de machucados na boca, emagrecimento significativo e escurecimento dos dentes. Em geral, a deterioração física ocorre em decorrência do uso compulsivo da substância, implicando em estado de esgotamento físico. Durante o consumo, as mulheres se abstêm dos cuidados básicos de saúde, alimentação, sono e higiene pessoal (Limberger et al., 2016). Percebe-se que essa população normalmente apresenta maiores prejuízos na saúde física em geral se comparada com indivíduos que não fazem uso de crack (Borders et al., 2009). Apesar da precariedade de recursos na saúde pública, há a necessidade dos serviços especializados estarem equipados para atender demandas básicas de cuidado físico e higiene (Kantorski, 2009).

Por fim, o quarto subtema ("Prostituição") está contemplado no tema "Comorbidades e consequências” por ter sido considerada pelos GFs como uma estratégia para obtenção da substância, ou seja, uma consequência do consumo do crack. Usualmente a prostituição é um recurso após a instauração da dependência como uma forma de manter o consumo (Caixieta et al., 2015; Guimarães et al., 2017). Sendo assim, intervenções devem ser planejadas com a compreensão da dinâmica da prostituição. Ressalta-se que um importante fator de risco no contexto da prostituição é o uso do crack durante a prática sexual, tornando as usuárias mais vulneráveis à violência sexual e a relações desprotegidas (Ribeiro \& Laranjeira, 2012; Vernaglia et al., 2017). A prostituição sob efeito de substâncias está fortemente relacionada à violência, o que gera a urgência de elaboração de estratégias preventivas, principalmente visando à diminuição da revitimização (Caixieta et al., 2015; Ribeiro \& Laranjeira, 2012).

\section{Atendimentos e serviços de saúde pública}

No eixo temático "Atendimento e serviços de saúde pública" foram incluídos temas sobre aspectos referentes à rede de saúde pública, aos atendimentos prestados pelos profissionais, aos desafios e limitações dos serviços, e aspectos que podem ser aprimorados nos locais de atuação (Tabela 2). O primeiro tema desse eixo foi denominado "Fluxo da rede", abrangendo descrições sobre como se dá, na percepção dos profissionais, o fluxo de encaminhamentos da rede de saúde e assistência para usuárias de crack. Ressalta-se que todos os GFs apontaram para uma baixa demanda de mulheres usuárias de crack para tratamento. A percepção do GF 1 foi de que o fluxo de encaminhamentos para os hospitais de desintoxicação funciona de forma rápida devido à menor procura de mulheres do que homens para atendimentos. O GF 2 explicitou o descontentamento com a burocracia que os serviços precisam enfrentar para solicitar os encaminhamentos 
necessários na rede de saúde. Já o GF 3 discutiu que para o fluxo ser efetivo seria necessária a integração de ações com a comunidade e com os familiares das pacientes. As diferentes percepções entres os GFs podem estar relacionadas à possibilidade de distinções nos procedimentos de encaminhamento em cada um dos serviços citados. Diante dessa hipótese, pode-se sugerir que os locais de atendimento aos usuários de substâncias, apesar de estarem baseados nas mesmas politicas públicas, não possuem atendimentos padronizados, evidenciando diferenças entre os serviços do Rio Grande do Sul e de São Paulo.

Tabela 2

Atendimento e serviços de saúde pública.

\begin{tabular}{|c|c|}
\hline Temas & Unidades de análise representativas \\
\hline Fluxo da rede & $\begin{array}{l}\text { "Geralmente mulher não tem problema em conseguir vaga. Geralmente em até } 72 \text { horas, } \\
\text { que seria assim o período que elas ficam na emergência, a vaga surge. Neste fluxo assim, } \\
\text { emergência, internação. Depois da internação que é feito para a rede” (GF 1). } \\
\text { "Eu passo vários dias sem atender nenhuma mulher, assim, às vezes eu passo uma } \\
\text { semana sem atender nenhuma mulher. . . nossa demanda de mulheres é mínima, } 15 \\
\text { leitos, um ocupado por mulher" (GF 1). } \\
\text { "Tem toda uma burocracia, pra não chamar de 'burrocracia' né, que tem que passar } \\
\text { por outro serviço, que tem que passar pelo posto de saúde, que tem que mandar pra } \\
\text { referência, contra referência” (GF 2). } \\
\text { "Um link imediato com outras pessoas formando uma rede de proteção para que esse } \\
\text { cuidado seja realmente efetivo" (GF 3). }\end{array}$ \\
\hline Manejo e intervenção & $\begin{array}{l}\text { “Porque se não tem o mínimo de criatividade não consegue trabalhar, até pela questão } \\
\text { da precarização dos recursos" (GF 2). } \\
\text { "Eu peguei e pensei, vou tentar ir para a rua, ver se assim direto, falando com aquelas } \\
\text { mais desintoxicadas e ver o que eu consigo fazer. E consegui trazer muitas" (GF 3). } \\
\text { "Como eu lido em relação ao acesso é garantir que o acesso seja livre e imediato. Então } \\
\text { todas as vezes que se rediscute acolhimento nem se cogita que o atendimento não seja } \\
\text { portas abertas... . O atendimento, a qualidade do atendimento ajuda também, pro } \\
\text { paciente não aderir ao tratamento por causa dos profissionais" (GF 3). }\end{array}$ \\
\hline Limitações e Desafios & $\begin{array}{l}\text { “Eu acompanhei a usuária até o hospital porque estava em trabalho de parto e eu } \\
\text { cheguei e a enfermeira falou: 'A então é você que me manda essas pessoas? você?'- isso } \\
\text { na frente da usuária. É muito triste” (GF 3). }\end{array}$ \\
\hline $\begin{array}{l}\text { Conhecimentos } \\
\text { necessários para o } \\
\text { manual }\end{array}$ & 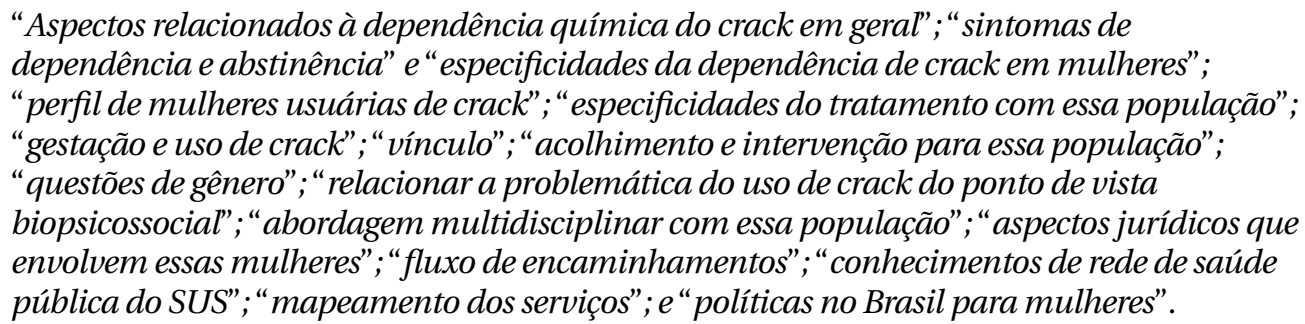 \\
\hline
\end{tabular}

Sabe-se que as mulheres usuárias de drogas buscam com menor frequência tratamento para problemas com drogas (Attilio et al., 2011). O atendimento e encaminhamentos necessários para as usuárias de crack devem ser realizados de forma rápida e eficiente, pois a agilidade auxilia na possibilidade de adesão ao tratamento (Balbinot et al., 2016). É de extrema importância a comunicação eficaz entre os serviços de saúde e assistência, respeitando a competência técnica de cada local (Paes, Schimith, Barbosa,
\& Righ, 2013). O excesso de burocracia no sistema de saúde público é um problema descrito na literatura. O número excessivo de regras e procedimentos para regular e formalizar as práticas nos serviços dificulta a efetividade do atendimento prestado. Por fim, ações estratégicas que visam o engajamento da família e da comunidade são veículos de construção de rede de apoio para tal população. Os serviços devem promover ações de reinserção social, visto que a maioria das 
mulheres não possuem rede de apoio para se manterem em tratamento (Ramiro et al., 2014).

No tema "Manejo e intervenção" foram identificadas descrições das intervenções para as usuárias. Nos três GFs foi percebido o empenho dos profissionais para promover ações e atividades de aproximação das mulheres usuárias de crack com o serviço e a busca do trabalho efetivo, mesmo com dificuldades recorrentes. Corroborando com tais resultados, um estudo identificou que os profissionais que atuavam com usuários de substâncias adotavam ações proativas, com desenvolvimento de atividades e projetos, mesmo sem recursos financeiros, pela motivação em investir na qualidade dos atendimentos (Horta et al., 2013). Percebe-se que, mesmo com a escassez de recursos, os profissionais conseguem desenvolver tarefas, intervenções e ações de cuidado, demonstrando abertura e acolhimento (Brasil, 2003; International Narcotics Control Board, 2017; Salles \& Silva, 2017).

No tema "Limitações e Desafios" foram observadas descrições dos profissionais sobre as adversidades vivenciadas nos serviços de saúde no atendimento a mulheres usuárias de crack. Todos os GFs apresentaram percepções semelhantes, como a prevenção na atenção básica, o aumento na motivação das usuárias para iniciar e manter-se em tratamento, a complexidade da gestante usuária, a motivação dos profissionais no atendimento dessa população e o combate ao preconceito. Entre as falas dos profissionais, destacou-se o relato de um participante sobre sentir-se impotente frente a esses desafios, como "Dom Quixote lutando contra o moinho de vento" (GF 2). A reprodução de estigmas sociais e julgamentos morais pelos profissionais contribuem para o afastamento das mulheres dos serviços de saúde (Ahern, Stuber, \& Galea, 2007; Costa \& Paiva, 2016; Limberger et al., 2015). Compreender questões relacionadas ao gênero pode auxiliar a redimensionar as dificuldades dessas usuárias na manutenção do tratamento, além de aumentar a motivação para atender essa população (Ferreira et al., 2015; Greenfield, Trucco, McHugh, Liconln, \& Gallop, 2007). O Plano Nacional de Políticas para Mulheres incentiva o desenvolvimento de estudos e pesquisas sobre desigualdade de gênero associado ao uso de substâncias psicoativas. Com isso, estimula a construção de estratégias de acolhimento que considerem especificidades de gênero e o sofrimento causado pelo uso de substâncias (Brasil, 2013). Outro desafio para os serviços de saúde é o cuidado com as gestantes usuárias de crack, considerando as complicações clínicas, além da maior vulnerabilidade emocional entre elas (Ribeiro \& Laranjeira, 2012). As características específicas da gravidez entre usuárias de crack incluem as dificuldades sociais, sintomatologias do transtorno por uso de substâncias, além de limitações e escassez de recursos dos serviços para atender adequadamente as demandas da gestação (Coles, Dartnall, \& Astbury, 2013).

No tema "Aprimoramento" foram selecionado estratos que descreviam o que os GFs indicaram em termos de melhorias nos serviços de saúde. Assim, apontou-se a necessidade de criação de um serviço de saúde específico para mulheres usuárias de substâncias, porém foi reconhecida a dificuldade da implementação em curto prazo. Como alternativa, foi sugerida a implementação de capacitações como estratégia de aprimoramento dos serviços, visando instrumentalizar os profissionais para lidar com as demandas. Por fim, os GFs indicaram que é necessária a oferta de melhores condições de trabalho para os profissionais. Reconhece-se a carência de espaços de saúde específicos para mulheres e a sua importância devido às diferentes demandas de gênero que envolvem as usuárias de crack (International Narcotics Control Board, 2017). Contudo, qualificar e aprimorar o acolhimento e a intervenção é uma prioridade, independentemente do modelo e do serviço em que os profissionais atuam (Greenfield et al., 2007; United Nations Office on Drugs and Crime, 2016).

A atenção básica foi apontada, pelos GFs, como a maior deficitária em conhecimentos sobre transtorno por uso de substâncias. A possibilidade de instrumentalizar a atenção básica pode trazer benefícios, com a implementação de estratégias de prevenção e manejo de casos de baixa complexidade, evitando encaminhamentos desnecessários para os demais níveis de complexidade (Brasil, 2003). A capacitação de profissionais, além de fornecer subsídios para manejo e intervenção no transtorno por uso de substâncias, também atua como foco de desestigmatização (Costa \& Paiva, 2016). Além da educação continuada, existem outras condições de trabalho que devem ser qualificadas. Dificuldades como baixos salários, atraso no pagamento, serviços de alta complexidade com baixos recursos e número excessivo de atendimentos ocasionam em sobrecarga emocional dos profissionais. Percebe-se, assim, que para além de ações de cuidado com as usuárias de crack, a promoção de 
saúde e estratégias de cuidado com os profissionais é essencial para uma atuação efetiva (Horta et al., 2013).

Por fim, no tema "Conhecimentos necessários para o manual” observou-se informações que, segundo os participantes, deveriam ser abordadas em um manual de conhecimentos específicos para profissionais que atuem com mulheres usuárias de crack. Os conteúdos indicados pelos GFs foram: características gerais (sintomas) do transtorno por uso de crack, fatores biopsicossociais, especificidades do consumo de crack entre mulheres, especificidades do tratamento, gestação das usuárias, vínculo, acolhimento, intervenção e informações sobre questões de gênero. Em relação a informações da rede e do tratamento foram sugeridos temas sobre abordagem multidisciplinar, incluindo aspectos jurídicos, fluxo de encaminhamentos, conhecimentos de rede de saúde pública do SUS, mapeamento dos serviços e políticas nacionais para mulheres usuárias de substâncias.

\section{Limitações e considerações finais}

A maior parte das políticas públicas no Brasil no contexto de uso de substância foi desenvolvida com base em estudos com a população masculina. Porém, é crescente o número de mulheres usuárias de drogas como o crack, ressaltando a necessidade de políticas públicas, estratégias de acolhimento e de intervenção que considerem as diferenças de gênero. Conhecer as percepções e crenças dos profissionais da saúde sobre mulheres usuárias de crack e sobre os serviços de saúde prestados a essa população específica pode auxiliar na elaboração de estratégias efetivas. Assim, destaca-se que o profissional de psicologia pode vir a ser uma ferramenta de suma importância para a modificação de crenças e estereótipos acerca das usuárias, fomentando um atendimento mais eficaz e humanizado no tratamento ao uso de substâncias.

Destacam-se algumas limitações em relação ao presente estudo. A existência de apenas um encontro nos GFs pode ser considerada uma limitação, impossibilitando que os Grupos se constituíssem de forma mais substancial, o que oportunizaria o aprofundamento da discussão. Também é importante salientar que o GF realizado em São Paulo teve condições diferentes dos demais. Tais diferenças se deram devido à necessidade de o GF ocorrer no local de trabalho de alguns dos participantes em São Paulo, não sendo um lugar neutro como ocorreu em Porto Alegre. Além disso, houve uma limitação do tempo que a instituição de trabalho cedeu aos profissionais para que participassem da pesquisa no GF de São Paulo. No entanto, todas as perguntas do roteiro foram respondidas igualmente e todos os GFs estavam engajados na tarefa, não indicando que o local interferiu no andamento do estudo.

Outra limitação a ser considerada é que alguns dos participantes dos GFs, tanto em Porto Alegre quanto em São Paulo, faziam parte da mesma rede de serviços. Também havia profissionais de diferentes áreas de formação e, em função de relações hierárquicas de trabalho, isto pode ter gerado um desconforto ou menor participação de alguns dos profissionais. No entanto, por meio dos relatos dos participantes, percebeu-se apreço pelo contexto do GF e pela possibilidade de trocar ideias e ouvir os colegas. Vale ressaltar que foi explicitado pelos profissionais o quanto os espaços de troca eram importantes e que, embora a finalidade do GF fosse a pesquisa, o conteúdo discutido era de extrema relevância para o dia a dia dos participantes

Apesar das limitações citadas, observa-se a relevância de o estudo ter sido composto por três GFs e por duas diferentes cidades, oportunizando observar as semelhanças nas percepções e experiências dos profissionais em relação às características dessas mulheres. É possível perceber que o preconceito, relacionado às mulheres usuárias de crack, contempla a sociedade como um todo, incluindo assim os próprios profissionais. Estes devem assumir um papel ativo para romper com o estigma social nos serviços e na comunidade onde atuam, o que ressalta a importância do fomento a práticas de educação continuada. Nesta perspectiva, é indicado o desenvolvimento de capacitações para auxiliar nos aspectos identificados no estudo, com melhores estratégias de prevenção, acolhimento e qualificação profissional, possibilitando o aprimoramento dos profissionais em diferentes níveis de complexidade. Sugere-se que estudos futuros possam desenvolver capacitações e materiais focados nessa temática como forma de auxiliar os profissionais e, assim, também qualificar o atendimento prestado para a população feminina usuária de crack. 
Maciel, L., Schneider, J. A., Chambart, D., Grassi-Oliveira, R., \& Habigzang, L. F. (2020). Percepções de profissionais sobre atendimento para usuárias de crack.

\section{Referências}

Ahern, J., Stuber, J., \& Galea, S. (2007). Stigma, discrimination and health of illicit drug users. Drug Alcohol Depend, 88(11), 188-196. https://doi.org/10.1016/j.drugalcdep.2006.10.014

Attilio, J. S., Rodrigues, F. P., Renovato, R. D., Sales, C. M., Alvarenga, R. M., Moreira, M. T.,... Pereira, N. C. A. (2011). Cobertura vacinal contra hepatite B entre usuários de drogas ilícitas. Acta Paulista de Enfermagem, 24(1), 101-106. https://doi.org/10.1590/S0103-21002011000100015

Balbinot, A. D., Horta, R. L., Soares J D. C., Brasil, R. A., Poletto, S., \& Teixeira, M.B. (2016). Hospitalizações por uso de drogas não se alteram com uma década de Reforma Psiquiátrica. Revista de Saúde Pública, 50: 1-9. https://doi.org/10.1590/S1518-8787.2016050006085

Barbosa, G. C., Cobayashi, K., \& de Oliveira, M. A. F. (2017). Atitudes dos trabalhadores de um centro de atenção psicossocial frente ao uso do álcool e alcoolismo. Revista Eletrônica de Graduação do UNIVEM, 10(1), 1-12. http://revista.univem.edu.br/REGRAD/article/view/1842

Bastos, F. I., \& Bertoni, N. (Orgs.). (2014). Pesquisa nacional sobre o uso de crack: quem são os usuários de crack e/ ou similares do Brasil? Quantos são nas capitais brasileiras? Rio de Janeiro, RJ: Fundação Oswaldo Cruz.

Bertoni, N., Burnett, C., Cruz, M. S., Andrade, T., Bastos, F. I., Leal, E., \& Fischer, B. (2014). Exploring sex differences in drug use, health and service use characteristics among young urban crack users in Brazil. International Journal for Equity in Health, 13(1), 70. https://doi.org/10.1186/s12939-014-0070-x

Borders, T. F., Booth, B. M., Falck, R. S., Leukefeld, C., Wang, J., \& Carlson, R. G. (2009). Longitudinal changes in drug use severity and physical health-related quality of life among untreated stimulant users. Addictive Behaviors, 34(11), 959-964. https://doi.org/10.1016/j.addbeh.2009.06.002

Brasil. (2003). A política do Ministério da Saúde para atenção integral a usuários de álcool e outras drogas. Brasília, DF: Ministério da Saúde. http://bvsms.saude.gov.br/bvs/publicacoes/pns_alcool_drogas.pdf

Brasil. (2005). Resolução no 3/gsipr/ch/conad, de 27 de outubro de 2005. Aprova a Política Nacional Sobre Drogas. Brasília, DF: Senad. http://www.justica.gov.br/central-de-conteudo/politicas-sobre-drogas/cartilhas-politicassobre-drogas/2011legislacaopoliticaspublicas.pdf

Brasil (2010). Decreto $n^{\circ}$ 7.179, de 20 de maio de 2010. Institui o Plano Integrado de Enfrentamento ao Crack e outras Drogas, cria o seu Comitê Gestor, e dá outras providências. Brasília, DF: Presidência da República. http://www.planalto.gov.br/ccivil_03/_ato2007-2010/2010/decreto/d7179.htm

Brasil. (2013). Plano Nacional de Políticas para as Mulheres. Brasília, DF: Secretaria de Política para Mulheres. http://www.spm.gov.br/assuntos/pnpm/publicacoes/ pnpm-2013-2015-em-22ago13.pdf

Braun, V., \& Clarke, V. (2006). Using thematic analysis in psychology. Qualitative Research in Psychology, 3(2), 77-101. https://doi.org/10.1191/1478088706qp063oa

Büchele, F., Coelho, E. B. S., \& Lindner, S. R. (2009). A promoção da saúde enquanto estratégia de prevenção ao uso das drogas. Ciência \& Saúde Coletiva, 14(1), 267-273. https://doi.org/10.1590/S1413-81232009000100033

Caixieta, F. C., Silva, Y. V., Lucchese, R., Felipe, R. L., Vera, I., \& Bueno, A. A. (2015). Vulnerabilidade de mulheres em uso e abuso de substâncias psicoativas. Investigação Qualitativa em Saúde, 1, 153-157.

Coles, J., Dartnall, E., \& Astbury, J. (2013). "Preventing the pain” when working with family and sexual violence in primary care. International Journal of Family Medicine, 2013, 1-7. https://doi.org/10.1155/2013/198578

Costa, P. H. A. D., \& Paiva, F. S. D. (2016). Revisão da literatura sobre as concepções dos profissionais de saúde sobre o uso de drogas no Brasil: modelo biomédico, naturalizações e moralismos. Physis: Revista de Saúde Coletiva, 26(3), 1009-1031. https://doi.org/10.1590/s0103-73312016000300015

Cruz, V. D., Oliveira, M. M., Pinho, L. B., Coimbra, V. C. C., Kantorski, L. P., \& Oliveira, J. F. (2014). Condições sociodemográficas e padrões de consumo de crack entre mulheres. Texto \& Contexto Enfermagem, 23(4), 1068-1076. https://doi.org/10.1590/0104-07072014000580013

Dualibi, L. B., Ribeiro, M., \& Laranjeira, R. (2008). Profile of cocaine and crack users in Brazil. Caderno de Saúde Pública, 24, 545-557. https:// doi.org/10.1590/S0102-311X2008001600007

Ferreira, A. C. Z., Borba, L. D. O., Capistrano, F. C., Czarnobay, J., \& Maftum, M. A. (2015). Fatores que interferem na adesão ao tratamento de dependência química: percepção de profissionais de saúde. Revista Mineira de Enfermagem, 19(2), 150-164. https://doi.org/10.5935/142762.20150032 
Gladwin, T. E., Figner, B., Crone, E. A., \&Wiers, R. W. (2011). Addiction, adolescence, and the integration of control and motivation. Developmental Cognitive Neuroscience, 1(4), 364-376. https://doi.org/10.1016/j.dcn.2011.06.008

Grassi-Oliveira, R., Pezzi, J. C., Daruy-Filho, L., Viola, T. W., Francke, I. D., Leite, C. E., \& Brietzke, E. (2012). Hair cortisol and stressful life events retrospective assessment in crack cocaine users. The American Journal of Drug and Alcohol Abuse, 38(6), 535-538. https://doi.org/10.3109/00952990.2012.694538

Greenfield, S. F., Trucco, E. M., McHugh, R. K., Lincoln, M., \& Gallop, R. J. (2007). The Women's Recovery Group Study: A stage i trial of women-focused group therapy for substance use disorders versus mixed-gender group drug counseling. Drug and alcohol dependence, 90(1), 39-47. https://doi.org/10.1016/j.drugalcdep.2007.02.009

Gueta, K. (2017). A qualitative study of barriers and facilitators in treating drug use among Israeli mothers: An intersectional perspective. Social Science \& Medicine, 187, 155-163. https://doi.org/10.1016/j.socscimed.2017.06.031

Guimarães, R. A., Castro, V. D. O. L., Stabile, A. C., Motta-Castro, A. R. C., Carneiro, M. A. S., Araujo, L. A.,... \& Teles, S. A. (2017). Gender differences in patterns of drug use and sexual risky behaviour among crack cocaine users in Central Brazil. BMC Psychiatry, 17(1), 412. https://doi.org/10.1186/s12888-017-1569-7

Horta, R. L., Esswein, G. C., \& Horta, C.L. (2013). Percepção de profissionais de saúde deCAPSI quanto a demandas relativas ao consumo de crack. Ciência e Saúde Coletiva, 18(4), 1099-1108. https://doi.org/10.1590/S1413-81232013000400023

InternationalNarcoticsControlBoard.(2017). ReportoftheInternationalNarcoticsControlBoardfor2016.NewYork: United Nations. https://www.incb.org/documents/Publications/AnnualReports/AR2016/English/AR2016_E_ebook.pdf

Kantorski, L. P., Jardim, V. R., Wetzel, C., Olschowsky, A., Schneider, J. F., Heck, R. M.,... Saraiva, S. S. (2009). Satisfação dos usuários dos centros de atenção psicossocial da região Sul do Brasil. Revista de Saúde Pública, 43(sup. 1), 29-35. https://doi.org/10.1590/S0034-89102009000800006

Kluwe-Schiavon, B., Viola, T. W., Sanvicente-Vieira, B., Pezzi, J. C., \& Grassi-Oliveira, R. (2016). Similarities between adult female crack cocaine users and adolescents in risky decision-making scenarios. Journal of Clinical and Experimental Neuropsychology, 38(7), 795-810. https://doi.org/10.1080/13803395.2016.1167171

Laranjeira, R., Madruga, C. S., Pinsky, I., Caetano, R., Ribeiro, M., \& Mitsuhiro, S. (2012). II Levantamento Nacional de Álcool e Drogas (Lenad): Uso de cocaína e crack no Brasil. São Paulo, SP: Inpad. http://inpad.org.br/lenad/ cocaina-e-crack/resultados--preliminares/

Laport, T. J., Costa, P. H. A., Mota, D. C. B., \& Ronzani, T. M. (2016). Percepções e práticas dos profissionais da atenção primária à saúde na abordagem sobre drogas. Psicologia: Teoria E Pesquisa, 32(1), 143-150. https://doi.org/10.1590/0102-37722016012055143150

Limberger, J., Nascimento, R. D. S. D., Schneider, J. A., \& Andretta, I. (2016). Women users of crack: systematic review of Brazilian literature. Jornal Brasileiro de Psiquiatria, 65(1), 82-88. https://doi.org/10.1590/0047-2085000000107

Limberger, J., Schneider, J. A., \& Andretta, I. (2015). Especificidades do tratamento de mulheres usuárias de crack: interface com direitos humanos. Psicologia em Pesquisa, 9(2), 13-147. https://doi.org/10.5327/Z1982-1247201500020004

Moraes, M. E. F., Roso, A., \& Lara, M. P. D. (2018). Gênero como uma categoria de análise nos estudos brasileiros sobre mulheres e consumo de crack. Gerais: Revista Interinstitucional de Psicologia, 11(1), 11-25. http://pepsic. bvsalud.org/scielo.php?script=sci_arttext\&pid=S1983-82202018000100003\&lng=pt\&nrm=iso

Oliveira, J. F. D., Paiva, M. S., \& Valente, C. L. (2006). Representações sociais de profissionais de saúde sobre o consumo de drogas: um olhar numa perspectiva de gênero. Ciência \& Saúde Coletiva, 11, 473-481. https://doi.org/10.1590/S1413-81232006000200024

Oliveira, P. S., Rodrigues, V. P., Morais, R. L. G. L., \& Machado, J. C. (2016). Assistência de profissionais de saúde à mulher em situação de violência sexual: revisão integrativa. Revista de Enfermagem UFPE, 10(5), 1828-1839. https://doi.org/10.5205/1981-8963-v10i5a13563p1828-1839-2016

Paes, L. G., Schimith, M. D., Barbosa, T. M., Righ, L. B. (2013). Rede de atenção em saúde mental na perspectiva dos coordenadores de serviços de saúde. Trabalho e Educação em Saúde, 11(2), 395-409. https://doi.org/10.1590/ S1981-77462013000200008

Pinto, V. M., Tancredi, M. V., Buchalla, C. M., \& Miranda, A. E. (2014). History of syphilis in women living with aids and associated risk factors in São Paulo, Brazil. Revista da Associação Médica Brasileira, 60(4), 342-348. https://doi.org/10.1590/1806-9282.60.04.013

Ramiro,F.S.,Padovani,R.C.,\&Tucci,A.M. (2014).Consumodecrackapartirdasperspectivas degêneroevulnerabilidade: uma revisão sobre o fenômeno. Saúde Em Debate, 38(101), 379-392. https://doi.org/10.5935/0103-1104.20140035 
Ribeiro, M., \& Laranjeira, R. (2012). O tratamento do usuário de crack. São Paulo: Artmed.

Salles, D. B., \& Silva, M. L. (2017). Percepção de profissionais da área de saúde mental sobre o acolhimento ao usuário de substância psicoativa em CAPSad. Cadernos Brasileiros de Terapia Ocupacional, 25(2), 341-349. https://doi.org/10.4322/0104-4931.ctoAO0803

Schneider, D. R., \& Lima, D. S. (2011). Implicações dos modelos de atenção à dependência de álcool e outras drogas na rede básica em saúde. Psico PUCRS, 42(2), 168-178. http://revistaseletronicas.pucrs.br/ojs/index.php/ revistapsico/article/viewFile/7153/6518

Secretaria Nacional de Políticas sobre Drogas. (2013). Estimativa do número de usuários de crack elou similares nas capitais do país. http://infograficos.estadao.com.br/especiais/crack/perfilusuarios.pdf

Silva, É. B. D. O., Pereira, A. L. D. F., \& Penna, L. H. G. (2018). Estereótipos de gênero no cuidado psicossocial das usuárias de cocaína e crack. Cadernos de Saúde Pública, 34(5), e00110317.

Smith, P. H., Homish, G. G., Leonard, K. E., \& Cornelius, J. R. (2012). Intimate partner violence and specific substance use disorders: findings from the national epidemiologic survey on alcohol and related conditions. Psychology of Addictive Behaviors, 26(2), 236-245. https://doi.org/10.1037/a0024855

Tractenberg, S. G., Viola, T. W., Rosa, C. S. O., Docati, J. M., Francke, I. D., Pezzi, J. C., Grassi-Oliveira, R. (2012). Exposição a trauma e transtorno de estresse pós-traumático em usuárias de crack. Jornal Brasileiro de Psiquiatria, 61(4), 206-213. https://doi.org/10.1590/S0047-20852012000400003

United Nations Office on Drugs and Crime. (2016). World drug report. New York. https://www.unodc.org/doc/ wdr2016/WORLD_DRUG_REPORT_2016_web.pdf

United Nations Office on Drugs and Crime. (2016). Guidelines on drug prevention and treatment for girls and women. New York. http://www.unodc.org/documents/drug-preventionandtreatment/unodc_2016_drug_prevention_ and_treatment_for_girls_and_women_E. pdf

Vernaglia, T. V. C., Leite, T. H., Faller, S., Pechansky, F., Kessler, F. H. P., Cruz, M. S., \& Group, B. C. (2017). The female crack users: Higher rates of social vulnerability in Brazil. Health Care for Women International, 38(11), 1170-1187. https://doi.org/10.1080/07399332.2017.1367001

\section{Luísa Maciel}

Psicóloga, Mestre em Psicologia pelo Programa de Pós-Graduação da Pontifícia Universidade Católica do Rio Grande do Sul, Porto Alegre - RS. Brasil.

E-mail: luisazmaciel@gmail.com

(D) https://orcid.org/0000-0002-0176-7655

\section{Jaluza Aimèe Schneider}

Psicóloga, Doutoranda em Psicologia pelo Programa de Pós-Graduação da Pontifícia Universidade Católica do Rio Grande do Sul, Porto Alegre - RS. Brasil.

E-mail: jaluza@hotmail.com

(1) https://orcid.org/0000-0001-7899-0786

\section{Daniela Chambart}

Psicóloga pela Pontifícia Universidade Católica do Rio Grande do Sul, Porto Alegre - RS. Brasil.

E-mail: danichambart@gmail.com

(D) https:/ / orcid.org/0000-0003-0268-0487

\section{Rodrigo Grassi-Oliveira}

Professor do Programa de Pós-Graduação em Psicologia da Pontifícia Universidade Católica do Rio Grande do Sul, Porto Alegre - RS. Brasil.

E-mail: rodrigo.grassi@pucrs.br

(D) https://orcid.org/0000-0001-9911-5921 


\section{Luísa Fernanda Habigzang}

Professora do Programa de Pós-Graduação em Psicologia da Pontifícia Universidade Católica do Rio Grande do Sul, Porto Alegre - RS. Brasil.

E-mail: luisa.habigzang@pucrs.br

(D) https://orcid.org/0000-0002-0262-0356

Os autores agradecem a todos os integrantes da associação Educadora São Carlos (AESC) do Hospital Mãe de Deus de Porto Alegre e do Centro de Referência de Álcool Tabaco e Outras Drogas (CRATOD) de São Paulo. Este estudo foi financiado pelo Conselho Nacional de Desenvolvimento Científico e Tecnológico (CNPq) [Número 466802/2014-5] e pela Secretária Nacional de Políticas sobre Drogas (SENAD) [Número 822647/2015].

Endereço para envio de correspondência:

Pontifícia Universidade Católica do Rio Grande do Sul (PUCRS). Av. Ipiranga, 6681, edifício 11/9ªndar/sala 924. CEP: 90619900. Porto Alegre - RS. Brasil.

Recebido 15/03/2018

Aceito 05/02/2019

Received 15/03/2018

Approved 05/02/2019

Recibido 15/03/2018

Aceptado 05/02/2019

Como citar: Maciel, L., Schneider, J. A., Chambart, D., Grassi-Oliveira, R., \& Habigzang, L. F. (2020). Percepções de Profissionais sobre Atendimentos em Saúde para Mulheres Usuárias de Crack. Psicologia: Ciência e Profissão, 40, 1-14. https://doi.org/10.1590/1982-3703003192955

How to cite: Maciel, L., Schneider, J. A., Chambart, D., Grassi-Oliveira, R., \& Habigzang, L. F. (2020). Perceptions of Professionals about Health Care for Women Crack Users. Psicologia: Ciência e Profissão, 40, 1-14.

https://doi.org/10.1590/1982-3703003192955

Cómo citar: Maciel, L., Schneider, J. A., Chambart, D., Grassi-Oliveira, R., \& Habigzang, L. F. (2020). Percepciones de Profesionales sobre Atenciones en Salud para Mujeres Usuarias de Crack. Psicologia: Ciência e Profissão, $40,1-14$. https://doi.org/10.1590/1982-3703003192955 